\title{
Research on the efficiency evaluation of communication network system via fuzzy analytic hierarchy process
}

\author{
Xu Yi-Qiong ${ }^{1}$, Feng Zhi-Yong ${ }^{1}$, Wang Bo ${ }^{2}$, Shi Lei ${ }^{3}$, Xue Yang ${ }^{3}$, Wei Jia-Hua ${ }^{3^{*}}$ \\ 1 School of Information and Communication Engineering, Beijing University of Posts and \\ Telecommunication, Beijing, 100876 \\ 2 Beijing JunChangShengAn Information Technology co., Itd, Beijing, 100095 \\ 3 Information and Navigation College, Air Force Engineering University, Shanxi Xi'An, 710073 \\ *Email: weijiahua@126.com
}

\begin{abstract}
Key words: communication network; efficiency evaluation; fuzzy analytic hierarchy process
Abstract: In this paper, the communication network system had been studied based on the schemes of efficiency evaluation. The relative assessment system and evaluation system had been designed via fuzzy analytic hierarchy process in terms of the timeliness, stability and security. Furthermore, the efficiency evaluation of a typical communication network system was performed by using of this novel proposal for the capability assessment system. Meantime, the calculation results are advised to verify the feasibility and rationality of the system.
\end{abstract}

\section{Introduction}

With the continuous development of science and technology, the communication network system are changing. Based on the rapid development of modern society and the high penetration of information, people pay more attention to the quality of the communication network system than before. Via some effectiveness evaluation methods, one can effectively measure the quality of communication network to provide a scientific basis for further construction of modern communication network system[1-4]. Therefore, it is of great theoretical and practical significance to in-depth study and solve the problem of communication network system effectiveness evaluation, which had become to a hot research direction in the field of communications.

So far, little progresses are obtained in the effectiveness evaluation of communication network, and most of them are belong to the communication network design stage of the technical problems, which is hindered the development reality network $[2,5]$. The communication network has the characteristics of large scale and complex structure. Moreover, the final system performance is decided by many factors, which have different effect[6]. The study to determine the weights of influence factor is of great significance for the accurate assessment of communication network efficiency via selecting the rational evaluation index system and using the scientific mathematics modeling method. Therefore, combining with actual usage and application of communication network system, we build a communication network system of effectiveness evaluation index system based on fuzzy hierarchy analysis method, and the fuzzy evaluation model is also established. The above results has the important reference value for similar system effectiveness evaluation research.

\section{The efficiency evaluation index system of communication network}

In the effectiveness evaluation process of communication network system, there are many influence factors, and the influence degree of each factor is difference. Therefore, the detailed analysis of communication network to choose the appropriate index collection is very important for constructing the effectiveness evaluation index system for communication network.

\section{The effectiveness evaluation system of communication network}

According to the preliminary theoretical research results and the characteristics of communication network system, a three-level effectiveness evaluation system of communication network is designed in this paper. The first level (target level) factor is the overall assessment target, which is communication network performance, the second level (criterion level) contains three disciplines: timeliness, stability and security, each factor in the criterion level contains a number of indicators. 


\section{The analysis of each indicator}

In the effectiveness evaluation index system for communication network, the overall factor $\mathrm{A}$ is the efficiency performance of communication network system. The three factors in the criterion level are expressed as $B_{i}(i=1,2,3)$, the third layer factors are given by $C_{i j}$, which is suitable for the $\mathrm{j}$-th factor in the $\mathrm{i}$-th two-level indicator. This section will show each indicator:

1) Communication timeliness $B_{1}$ has five indicators: communication coverage $C_{11}$, communication network delay-time $\mathrm{C}_{12}$, communication error rate $\mathrm{C}_{13}$, communication speed $\mathrm{C}_{14}$ and communication capacity $\mathrm{C}_{15}$. Communication coverage $\mathrm{C}_{11}$ is the spatial scope of communication support ability, which is a basic index. Communication network delay-time $\mathrm{C}_{12}$ is the required time for sending some information from the sender to the receiver. In the process of communication, because of that the influence of interference and noise would introduce some error, this part can be described by communication error rate $\mathrm{C}_{13}$. Communication speed $\mathrm{C}_{14}$ is used to characterize the rate of the information transmission. The size of the communication network data transmission bandwidth is shown as communication capacity $\mathrm{C}_{15}$, which is an efficiency index of the communication network system.

2) Communication security $B_{2}$ contains three evaluation indexes: information confidential $C_{21}$, anti-interference ability $\mathrm{C}_{22}$ and electromagnetic shielding ability $\mathrm{C}_{23}$. In order to reduce the search, intercept, deception and invasion of non-business parties to the communication network system, the ability of network system to intercept and anti-cheat resistance can be called information confidential $\mathrm{C}_{21}$. The ability of effective transmission of information after the disturbance performed on the communication network is considered as anti-jamming capability $\mathrm{C}_{22}$. At present, all kinds of electromagnetic interference and attack for communication network require higher protection, electromagnetic protection $\mathrm{C}_{23}$ has important influence on its safety, electromagnetic protection includes electromagnetic concealment and resisting electromagnetic to destroy.

3) Communication stability $B_{3}$ contains three evaluation factors: the continuous working ability $C_{31}$, network topology $C_{32}$ and network survivability $C_{33}$. Continuous working ability $C_{31}$ refers to the trouble-free working duration of the communication network system, the indicators need some statistical correlation within a long time working hours to determine the concrete values. The network topology $\mathrm{C}_{32}$ can determine the overall communication ability damage situation when some nodes cannot work normally, the topology of the reasonable system for communication network is very important. In the practical work process, the network system will be affected by all kinds of disturbances, eavesdropping and attack, network survivability $\mathrm{C}_{33}$ is expressed the ability of stable work of communication network in the above cases.

\section{The determining of relative weights}

Table 1 The Satty standard to construct judgment matrix

\begin{tabular}{cl}
\hline importance value & \multicolumn{1}{c}{ meaning } \\
\hline 1 & the latter and former are equally important. \\
5 & the latter are more important than the former. \\
7 & the latter are more important strongly than the former \\
9 & the latter are more important obviously than the former. \\
the latter are more important extremely than the former. \\
the middle of the state \\
if the ratio of factors $(i, j)$ is $a_{\mathrm{ij}}$, thus the one of factors $(j, i)$ \\
is $1 / a_{\mathrm{ij}}$
\end{tabular}

In the effectiveness evaluation system of communication network, the first and second level contain some items, and these items for overall effectiveness evaluation influence each are not identical, so that the relative weights need to be determined. In this paper, the Satty scale method is adopted to evaluate the indexes between the relative importance, shown as Table 1.

Based on the judgment standards, one can obtained the follow matrix $a$ :

$$
a-\left(\begin{array}{ccccc}
1 & a_{12} & a_{13} & \cdots & a_{1 n} \\
a_{21} & 1 & a_{23} & \cdots & a_{2 n} \\
a_{31} & a_{32} & 1 & \cdots & a_{3 n} \\
\vdots & \vdots & \vdots & \ddots & \vdots \\
a_{n 1} & a_{n 2} & a_{n 3} & \cdots & 1
\end{array}\right)
$$

By using of the addition-multiplication, the weights can be expressed as $w_{\mathrm{a}}=\left[\mathrm{w}_{1}, \mathrm{w}_{2}, \cdots, \mathrm{w}_{\mathrm{n}}\right]$ : 


$$
w_{i}(i=1,2, \cdots, n)=\frac{\sum_{j=1}^{n}\left(a_{i j} / \sum_{i=1}^{n} a_{i j}\right)}{\sum_{i=1}^{n} \Sigma_{j=1}^{n}\left(a_{i j} / \Sigma_{i=1}^{n} a_{i j}\right)}
$$

For example by the target level, based on the Satty judgment standards, the judgment matrix of communication timeless $\mathrm{B}_{1}$ :

$$
D\left(B_{1}\right)=\left(\begin{array}{ccccc}
1 & 2 & 3 & 1 / 2 & 2 \\
1 / 2 & 1 & 1 / 2 & 4 & 1 \\
1 / 3 & 2 & 1 & 5 & 1 / 2 \\
2 & 1 / 4 & 1 / 5 & 1 & 2 \\
1 / 2 & 1 & 2 & 1 / 2 & 1
\end{array}\right)
$$

Furthermore, one can obtain $w\left(E_{1}\right)=\left[\begin{array}{llll}0.23, & 0.12,0.10,0.44, & 0.11\end{array}\right]$.

\section{The efficiency evaluation model of communication network}

Based on the theory of fuzzy mathematics, the concrete processes to evaluate effectiveness of communication network system can be divided into five steps: One, set up the evaluation factor set; Two, construct the corresponding evaluation set; Three, Calculate index weight set; Four, determine the fuzzy membership degree; Five, evaluate comprehensively. The first and third steps in the construction of evaluation system has been completed, the second, fourth and fifth are the main points of the evaluation model. This section will present the realization method of effectiveness evaluation of communication network system in detail:

1) Set up the assemble of evaluation factors. In this paper, the evaluation factor set is composed of all the indicators in effectiveness evaluation index system for communication network. From the evaluation index system, one can find that the communication network effectiveness evaluation index system has 11 three-level index, which belong to the three criterion- level factors, respectively. Furthermore, these three criterion-level index factors form the target layer index.

2) Construct the corresponding evaluation set. The evaluation set is a relatively broad concept. Based on the collection in particular the evaluation grades of evaluation index and the actual communication network system, this paper selected evaluation set as "excellent, good, medium and poor", which took the evaluation sets:

$$
\mathrm{p}=\left[\mathrm{p}_{1}, \mathrm{p}_{2}, \mathrm{p}_{3}, \mathrm{p}_{4}\right]
$$

Here $\sum_{i=1}^{n} p_{i}=1$. If the evaluation for communication coverage $C_{11}$ communication timeliness $\mathrm{B}_{1}$ is "28\% excellent, $14 \%$ good, $28 \%$ medium and $30 \%$ poor", the corresponding evaluation is:

$$
\mathrm{P}\left(\mathrm{C}_{11}\right)=[0.28,0.14,0.28,0.14]
$$

3) Calculate index weight set. One can use the Satty scale method to get the corresponding judgment matrix, and calculate the index weight set. The calculation process is shown as Eqs. (1) and (2). Through this step, one can get the target layer index weight $w_{A}$ and rule layer index weight $\left\{w\left(B_{1}\right), w\left(B_{2}\right), w\left(B_{3}\right)\right\}$.

4) For the third level indexes, based on the evaluation set "excellent, good, medium and poor" evaluation set, one can obtain the fuzzy matrix to line the indicators evaluation classification. For the five tertiary index of communication timeliness $B_{1}$, through questionnaire survey and expert scoring method, one can get the evaluation sets of each indexes:

$$
\begin{aligned}
& \mathrm{p}\left(\mathrm{C}_{11}\right)-[0.28,0.14,0.28,0.30] \quad \mathrm{p}\left(\mathrm{C}_{12}\right)=[0.26,0.24,0.23,0.27] \\
& p\left(C_{13}\right)=[0.18,0.36,0.36,0.08] \quad p\left(C_{14}\right)=[0.46,0.12,0.21,0.21] \\
& \mathrm{p}\left(\mathrm{C}_{15}\right)=\left[\begin{array}{llll}
0.34, & 0.26, & 0.18, & 0.22
\end{array}\right]
\end{aligned}
$$

thus the fuzzy matrix of communication timeliness $\mathrm{B}_{1}$ can be expressed as:

$$
p\left(B_{1}\right)-\left(\begin{array}{llll}
0.28 & 0.14 & 0.28 & 0.30 \\
0.26 & 0.24 & 0.23 & 0.27 \\
0.18 & 0.36 & 0.38 & 0.08 \\
0.46 & 0.12 & 0.21 & 0.21 \\
0.34 & 0.26 & 0.18 & 0.22
\end{array}\right)
$$

Similar to the same process, the fuzzy matrixes $\left\{p\left(B_{2}\right), p\left(B_{3}\right)\right\}$ of communication security $B_{7}$ and communication stability $\mathrm{B}_{3}$ can be obtained.

5) Through the third step, one can get theinternal weights, namely, $\left\{w(A), w\left(B_{1}\right), w\left(B_{2}\right), w\left(B_{3}\right)\right\}$. Based on the fourth step, one can get fuzzy matrixes $\left\{\mathrm{p}\left(\mathrm{B}_{1}\right), \mathrm{p}\left(\mathrm{B}_{2}\right), \mathrm{p}\left(\mathrm{B}_{3}\right)\right\}$. Thus, from the formula for computing the fuzzy evaluation, one can get the evaluation result the criterion-level index:

$$
\bar{p}\left(B_{\mathrm{i}}\right)=w\left(B_{\mathrm{i}}\right) * p\left(B_{\mathrm{i}}\right) \quad i=1,2,3
$$

Meanwhile, the fuzzy matrix $\mathrm{P}(\mathrm{A})$ for the performance of communication network can be expressed as: 


$$
\mathrm{p}(\mathrm{A})=\left[\bar{p}\left(B_{1}\right), \bar{p}\left(B_{2}\right), \bar{p}\left(B_{3}\right)\right]^{\mathrm{T}}
$$

According to the judge matrix $\mathrm{D}(\mathrm{A})$ and the weights $\mathrm{w}(\mathrm{A})$, one can find the evaluation result of communication network:

$$
\bar{p}(A)=w(A) * \bar{p}(A)
$$

From the matrix $\bar{p}(A)$, we can get the evaluation result.

\section{Concrete Simulation}

In this section, we will select the communication network system of "Red Arrow 2017" action as an example to evaluate effectiveness by using of the communication system effectiveness evaluation index system constructed in this paper. Accordingly to the assessment results of communication network system, and some works can be done to increase the efficiency of the communication system. Specific system effectiveness evaluation implementation are presented as follow:

Step 1: Construct communication system effectiveness evaluation index system and set up the assembly of evaluation factors. In this paper, the constructed index system is shown in Section 2, all the indicators belong to the assembly of evaluation factors.

Step 2: The evaluation set is established. In this paper, the choice of evaluation set as "excellent, good, medium and bad".

Step 3: Calculate the index weight set of the criterion-level and index-level matrix $\left[\mathrm{D}(\mathrm{A}), \mathrm{D}\left(\mathrm{B}_{1}\right), \mathrm{D}\left(\mathrm{B}_{2}\right), \mathrm{D}\left(\mathrm{B}_{3}\right)\right]$. Based on Satty scale, using the method of questionnaire survey, one can get the corresponding judge matrixes. $D\left(B_{1}\right)$ is given by Eq. (3).

$$
D(A)=\left(\begin{array}{ccc}
1 & 3 & 5 \\
1 / 3 & 1 & 2 \\
1 / 5 & 1 / 2 & 1
\end{array}\right) D\left(B_{2}\right)=\left(\begin{array}{ccc}
1 & 1 / 2 & 4 \\
2 & 1 & 7 \\
1 / 4 & 1 / 7 & 1
\end{array}\right) D\left(B_{3}\right)=\left(\begin{array}{ccc}
1 & 1 / 3 & 2 \\
3 & 1 & 1 / 6 \\
1 / 2 & 6 & 1
\end{array}\right)
$$

Based on Eqs. (1) and (2), the weights $\left\{w(A), w\left(B_{1}\right), w\left(B_{2}\right), w\left(B_{3}\right)\right\}$ of the criterion-level and index-level are expressed as:

$$
\begin{aligned}
& w(A)=[0.52,0.27,0.21\rceil \quad w\left(B_{1}\right)=\lceil 0.23,0.12,0.10,0.44,0.11\rceil \\
& w\left(B_{2}\right)=[0.32,0.56,0.12] \quad w\left(B_{3}\right)=[0.22,0.65,0.13]
\end{aligned}
$$

Step 4: Obtain the fuzzy matrixes $\left\{p\left(B_{1}\right), p\left(B_{2}\right), p\left(B_{3}\right)\right\}$ for the third-level factors. $p\left(B_{1}\right)$ is given by Eq. (6).

$$
p\left(B_{2}\right)=\left(\begin{array}{llll}
0.23 & 0.36 & 0.32 & 0.11 \\
0.36 & 0.35 & 0.16 & 0.13 \\
0.46 & 0.28 & 0.18 & 0.08
\end{array}\right) \quad p\left(B_{3}\right)=\left(\begin{array}{llll}
0.41 & 0.35 & 0.15 & 0.09 \\
0.52 & 0.26 & 0.20 & 0.02 \\
0.26 & 0.36 & 0.28 & 0.10
\end{array}\right)
$$

Step 5: From Eqs. (7) and (8), we can get that

$$
p(A)=\left(\begin{array}{llll}
0.46 & 0.26 & 0.16 & 0.12 \\
0.36 & 0.30 & 0.20 & 0.04 \\
0.22 & 0.46 & 0.26 & 0.06
\end{array}\right)
$$

Furthermore, the assessment result are expressed as:

$$
\bar{p}(A)=w(A) * \bar{p}(A)=[0.44,0.24,0.18,0.06]
$$

Hence, one can get the total assessment result for communication network of "Red Arrow 2017" is excellent. Through $\mathrm{p}$ (A) value in Eq. (13), it can be found that the evaluation results of communication timeliness $B_{1}$ and communication security $B_{2}$ are both excellent, but communication stability $\mathrm{B}_{3}$ is good. Although the whole communication network system effectiveness evaluation result is good, it is necessary to strengthen the construction of the system stability to further increase the efficiency of the system.

\section{Conclusion}

In summary, the actual usage and application characteristics of communication network system are analyzed. Then the relative assessment system and evaluation system had been designed via fuzzy analytic hierarchy process in terms of the timeliness, stability and security. Through the concrete simulation of the communication network in "Red Arrow 2017" action, one can find that the validity and rationality of this method is verified. Furthermore, this method can be extended to the similar system of effectiveness evaluation process, the evaluation method for systems effectiveness evaluation research has the important reference value for similar system effectiveness 
evaluation research.

\section{References:}

[1] JI Xiang, SUN Qing-sheng. Study of Effectiveness Evaluation Based on ADC and AHP Model. Information and Technology, 2014, 5( 3) :20-26.

[2]Xue Yang. Research on the efficiency evaluation via fuzzy nalytic hierarchy process[J]. New Information, 2010, 4(6): 54-56.

[3] Guo Zhenkun. Effective Evalution of Communication System Based on Hiberarchy Analytical Mathed [J]. New Technology, 2016, 6(4): 37-44.

[4] Zhang Hao, Lu Yu. Effectiveness Evaluation for Communication System via AHP[J]. Communications Technology, 2009, 10: 66-68.

[5] Yao Min, Wang Dong. Effectiveness Evaluation Model for Troposcatter Communication System Based on AHP [J]. Radio Communications Technology, 2016, 2( 3) :26-28.

[6] Shi Lei. Efficiency evaluation of communication network system[J]. Radio Communications Technology, 2016, 1(2) :12-16. 